\title{
Ganglioglioma in the Third Ventricle: A Case Report and Literature Review
}

\author{
Nayuta Higa, ${ }_{1}$ Hajime Yonezawa, ${ }^{1}$ Tatsuki Oyoshi, ${ }^{1}$ Tsubasa Hiraki, ${ }^{2}$ Hirofumi Hirano, ${ }_{1}^{1}$ and Kazunori Arita ${ }^{1}$
}

\begin{abstract}
Gangliogliomas typically arise in the cerebral hemispheres, but may occur rarely in the ventricles. Herein, we report a 38-year-old woman who was treated for hydrocephalus caused by a ganglioglioma of the third ventricle. Magnetic resonance imaging (MRI) revealed a heterogeneously enhanced tumor occupying the anterior part of the third ventricle. A left trans-lateral ventricular trans-foramen of Monroi approach was effective in achieving subtotal resection of the tumor, which had arisen from the medial part of left thalamus to the hypothalamus. Follow-up MRI showed no recurrence of the tumor 5-years after surgery. On pathological examination, the tumor was composed of a glial component that presented features mimicking pilocytic astrocytoma with proliferations of large gangliocytic cells that stained positive for neuronal markers. A review of six similar cases in the literature, including our own, revealed hydrocephalus to be the main symptom of gangliogliomas, with pituitary insufficiencies and visual disturbances having also been reported. In conclusion, we highlight the importance of gangliogliomas in the differential diagnosis of third ventricular tumors presenting with hydrocephalus.
\end{abstract}

Keywords: ganglioglioma, third ventricle, hydrocephalus

\section{Introduction}

Gangliogliomas are rare, benign tumors of the central nervous system that primarily affect children and young adults. ${ }^{1)}$ Pathologically, they present as mixed neurogliogenic tumors composed of neoplastic proliferations of two types of cells, ganglion cells and glial cells. ${ }^{1-3)}$ Gangliogliomas most commonly arise in the cerebral hemispheres. Within this location, the temporal lobe, either alone or in combination with the frontal or parietal lobes, is preferentially affected. ${ }^{1-4)}$ Gangliogliomas are also known to occur in the thalamus, pineal gland, and cerebellum, but have rarely been reported in the third ventricle. ${ }^{1-3)}$ In this case report, we describe a 38-year-old woman who presented with hydrocephalus caused by a ganglioglioma in the third ventricle and review similar cases in the literature.

\footnotetext{
${ }^{1}$ Department of Neurosurgery, Graduate School of Medical and Dental Sciences, Kagoshima University, Kagoshima, Japan

${ }^{2}$ Department of Human Pathology, Field of Oncology, Graduate School of Medical and Dental Sciences, Kagoshima University, Kagoshima, Japan
}

Received: February 2, 2016; Accepted: February 29, 2016

\section{Case Report}

A 38-year-old Filipino-Japanese woman who had suffered from chronic headaches for the past month underwent magnetic resonance imaging (MRI) that revealed a third ventricular mass and obstructive hydrocephalus. On admission, the patient was neurologically intact but had complained of headaches, nausea, insomnia, and a low-grade fever. Blood levels of anterior pituitary hormones, human chorionic gonadotropin, and alpha-fetoprotein were within normal limits. A pre-operative computed tomography (CT) scan showed a tumor (35 $\mathrm{mm}$ in diameter) with partial calcification and multiple microcysts occupying the anterior part of the third ventricle and obstructing the foramen of Monroi (Fig. 1a). The tumor had low-signal intensity on $\mathrm{T}_{1}$-weighted MRI (Fig. 1b) and high-signal intensity on $\mathrm{T}_{2}$-weighted MRI (Fig. 1c). Gadolinium-enhanced MRI in the axial (Fig. 1d), coronal (Fig. 1e), and sagittal (Fig. 1f) planes showed heterogeneous tumor contrast enhancement, suggesting the presence of necrotic regions. Under a pre-operative diagnosis of high-grade glioma, pilocytic astrocytoma, or chordoid glioma, the tumor was subtotally resected via a left trans-lateral ventricular trans-foramen of Monroi approach except for a tiny portion that had attached to the left fornix. On surgical observations, a layer of ependymal tissue was seen covering the tumor. The tumor texture was relatively soft, and bleeding was minimal. The tumor appeared to have arisen from the medial part of the left thalamus to the hypothalamus. Although the patient developed transient diabetes insipidus followed by hyponatremia, the post-operative course was essentially smooth. The patient was discharged 2 weeks after surgery without any neurological deficits or hormonal imbalance. Post-operative gadolinium-enhanced MRI in the axial (Fig. 2a), coronal (Fig. 2b), and sagittal planes (Fig. 2c) showed complete disappearance of the tumor.

On pathological examination, the tumor had a biphasic appearance characterized by alternating densely fibrillated and loosely textured regions with abundant microcysts (Fig. 3a). No mitoses were observed. The proliferating cells had round or ovoid nuclei with moderate atypia and elongated hair-like thin cytoplasmic processes (Fig. 3b). Abundant eosinophilic granular bodies and Rosenthal fibers were apparent (Fig. 3c), and an area of calcification was observed (Fig. 3d). Immunostaining demonstrated positivity for glial fibrillary acidic protein (Fig. 4a), S-100 protein (Fig. 4b), and vimentin (Fig. 4c), but negativity for epithelial membrane antigen (Fig. 4d). The Ki-67 proliferative index was $0.9 \%$. These histological findings were initially suggestive of pilocytic astrocytoma. However, close detailed examination 

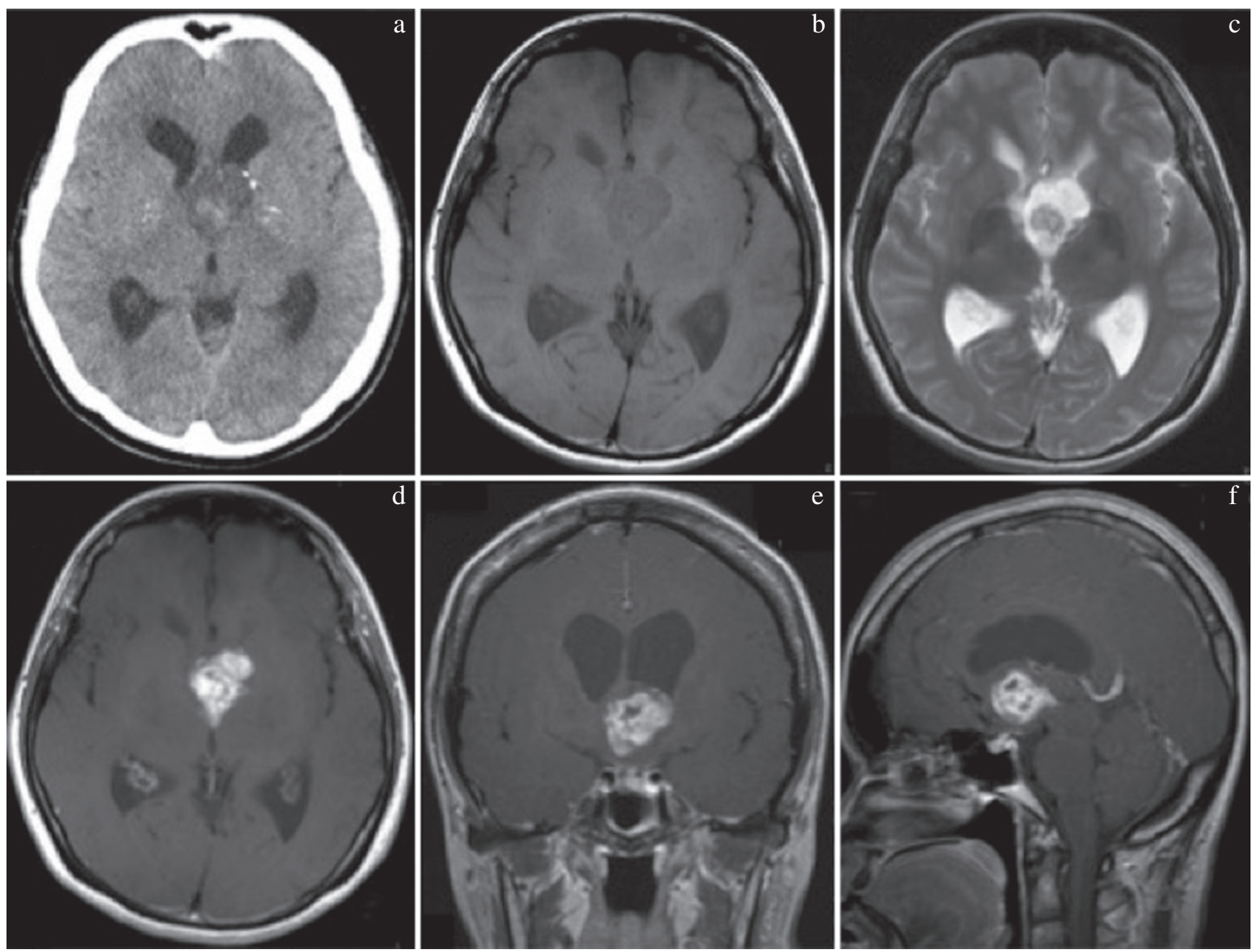

Fig. 1 Pre-operative computed tomography scan (a); $\mathrm{T}_{1}$-weighted (b), and $\mathrm{T}_{2}$-weighted (c) axial magnetic resonance imaging (MRI); and gadoliniumenhanced MRI in the axial (d), coronal (e), and sagittal (f) planes showing the presence of a tumor in the anterior part of the third ventricle causing hydrocephalus.

revealed potentially proliferating multinucleated or large cells (Fig. 5a) that stained positive for synaptophysin (Fig. 5b), and neuronal specific nuclear protein, NeuN (Fig. 5c), but negative for glial fibrillary acidic protein (Fig. 5d). These findings led to a final histopathological diagnosis of ganglioglioma (World Health Organization [WHO] grade I) with astrocytic proliferation. BRAF V600E mutation status was assessed by VE1 immunohistochemistry, but staining was not detected in the neuronal or glial components (Fig. 6).

Over the 5-year follow-up after surgery, the patient has remained recurrence-free and has been active in daily life, without neurological deficits.

\section{Discussion}

Gangliogliomas are rare, benign tumors that account for $0.3-1.3 \%$ of all intracranial tumors. ${ }^{1)}$ The vast majority of ganglioglioma patients (75-100\%) present with seizures, followed by topical symptoms relating to the anatomic site at which the tumor develops. ${ }^{1,2)}$ These tumors preferentially arise in the temporal lobe of the cerebral cortex but they may also occur in any other region of the central nervous system including, in rare instances, the third ventricle. Before the 1930s, there were only two published reports on gangliogliomas in or around the third ventricle. ${ }^{5,6)}$ Because of the inherent ambiguity of descriptions in the location and extent of the tumors, owing to the lack of modern neuroimaging techniques, we omitted these historic cases from our current review. As few as six cases, involving three men and three women (including our own), have been reported in the English literature in the CT and MRI era (Table 1). ${ }^{1,2,7)}$ The mean age of these patients was 31 years (range, 15-52 years), a little older than ganglioglioma patients with tumors in other regions. The most commonly reported symptoms were headaches and nausea caused by obstructive hydrocephalus. Visual disturbances and symptoms of pituitary insufficiencies, including general fatigue and diabetes insipidus, were also observed. No recurrence of the tumors was recorded during a mean follow-up of 40 months (range, 8-72 months).

Gangliogliomas reportedly present with various densities on CT scans (low density [38\%], isodensity [15\%], high density 

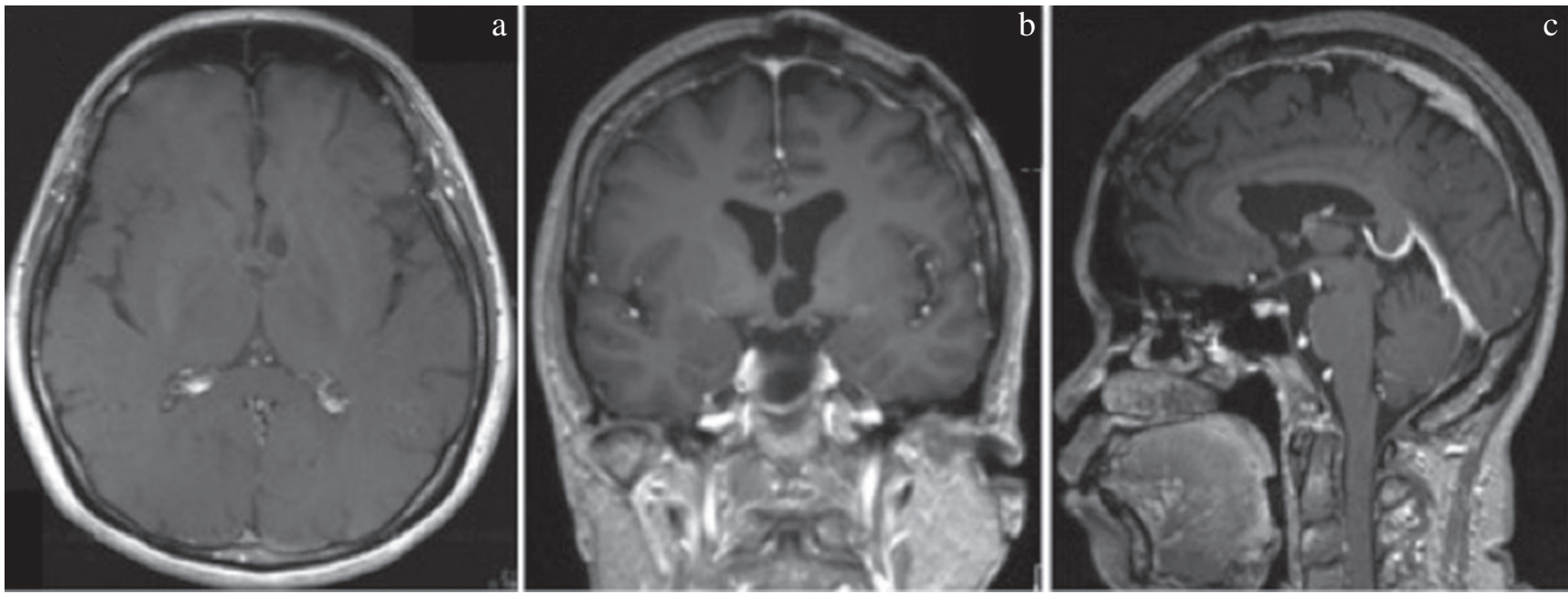

Fig. 2 Post-operative gadolinium-enhanced magnetic resonance imaging in the axial (a), coronal (b), and sagittal (c) planes showing complete disappearance of the tumor.

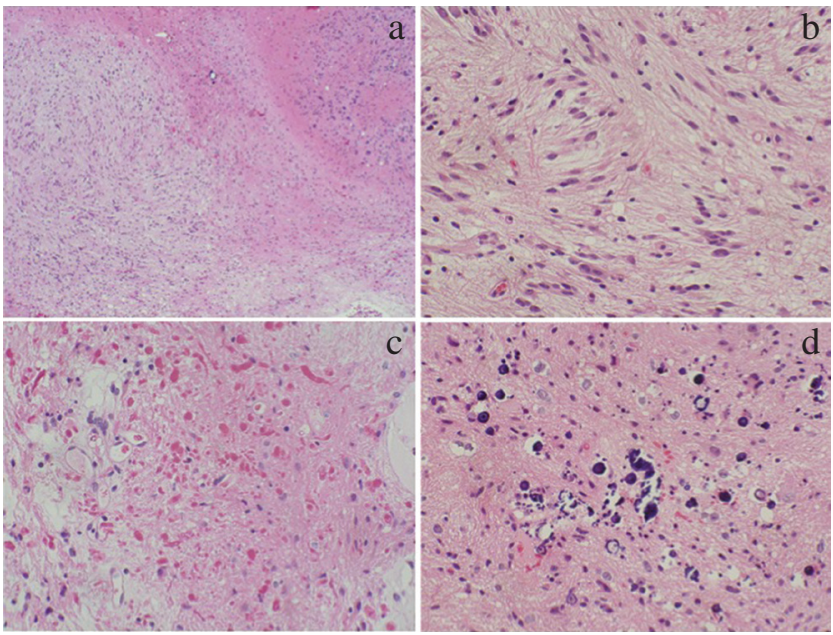

Fig. 3 Photomicrographs of the surgical specimen with hematoxylin and eosin staining demonstrating a biphasic pattern of texture (a), long bipolar cells (b), rich eosinophilic granular bodies and Rosenthal fibers (c), and an area of calcification (d).

[15\%], and mixed density [32\%]). ${ }^{3)}$ Calcification is observed in $20-83 \%$ of cases. ${ }^{1,3,8)}$ In general, the tumors are well enhanced and present mainly as isointense to hypointense lesions on $\mathrm{T}_{1}$-weighted MRI, and isointense to hyperintense lesions on $\mathrm{T}_{2}$-weighted MRI. ${ }^{1,48}$ Owing to their non-specific presentations on neuroimaging investigations, it can be difficult to differentiate gangliogliomas in the third ventricle from other types of tumors, pre-operatively. This was especially true in our patient in which the presence of a heterogeneous, irregular ring-like enhancement was suggestive of a malignant glioma. Advanced MRI techniques such as magnetic resonance spectroscopy, apparent diffusion coefficient mapping, and perfusion imaging might have led to the correct diagnosis of a benign tumor. Unfortunately, we were unable to perform these investigations because of the hydrocephalic nature of the patient's symptoms, which urged us to perform early surgery.

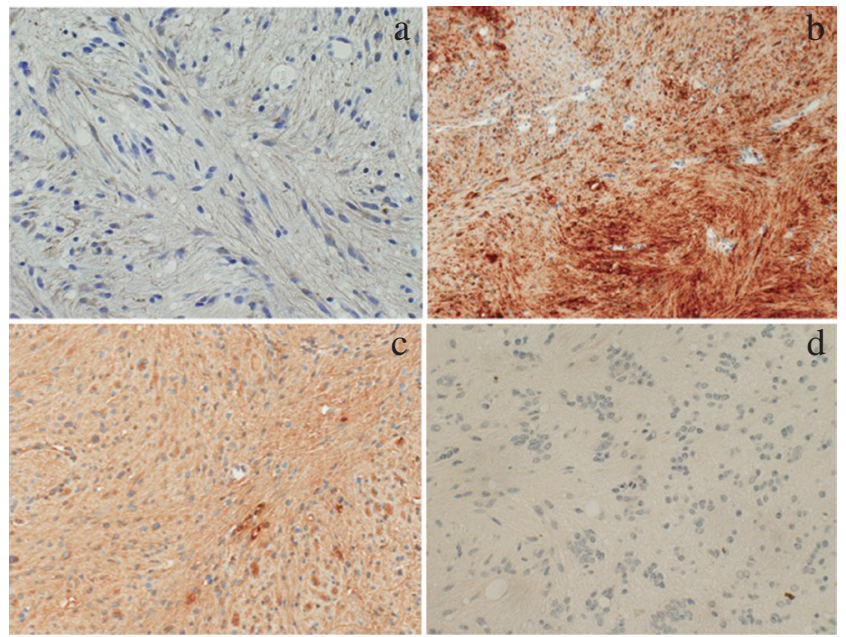

Fig. 4 Immunostaining of the surgical specimen demonstrating positivity for glial fibrillary acidic protein (a), S-100 protein (b), and vimentin (c), but negativity for epithelial membrane antigen (d).

Because gangliogliomas are indolent neuroglial tumors, their prognosis is largely dependent on the degree of resection. In general, 4-year survival rates have been reported to be at $100 \%$ in cases with total resection and $75 \%$ in cases with subtotal resection. ${ }^{3)}$ However, gangliogliomas may follow a course of malignant transformation to anaplastic gangliogliomas (WHO grade III) or glioblastomas (WHO grade IV). ${ }^{9)}$ Therefore, it is important to perform total resection of the tumor, where possible, for prolonged survival. In our patient, we achieved almost total resection of the tumor except for a tiny portion that had adhered to the left fornix. Despite the not observing any residue or recurrence on MRI, at 5-years after surgery, regular follow-ups will be mandatory for long-term survival.

Gangliogliomas are composed of proliferations of large ganglial cells and astrocytes with atypia. Ganglionic cells 


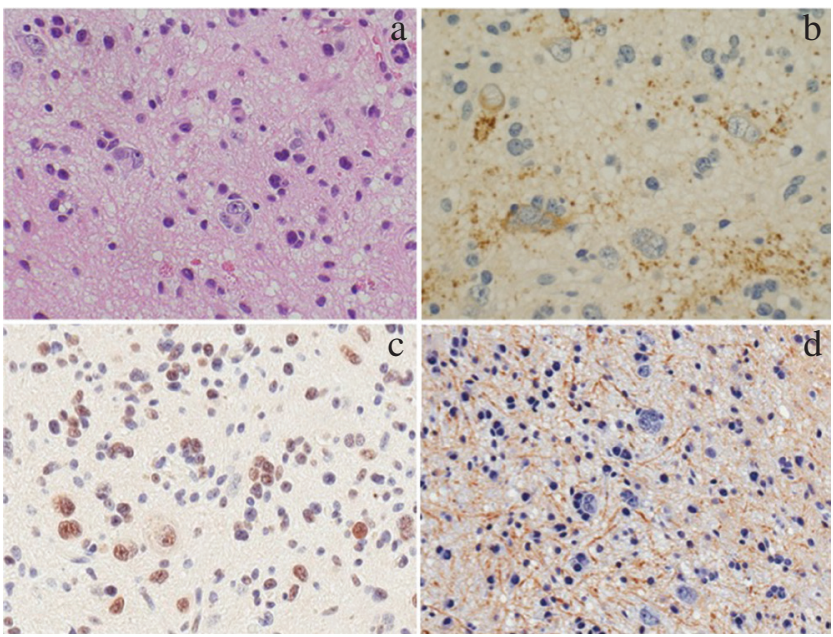

Fig. 5 Photomicrographs of the ganglionic-cell component showing, on hematoxylin and eosin staining, large cells and multinucleated cells scattered against dense fibrillary networks (a). Immunostaining of these cells demonstrated positivity for synaptophysin (b), and neuronal specific nuclear protein, NeuN (c), but negativity for glial fibrillary acidic protein (d).

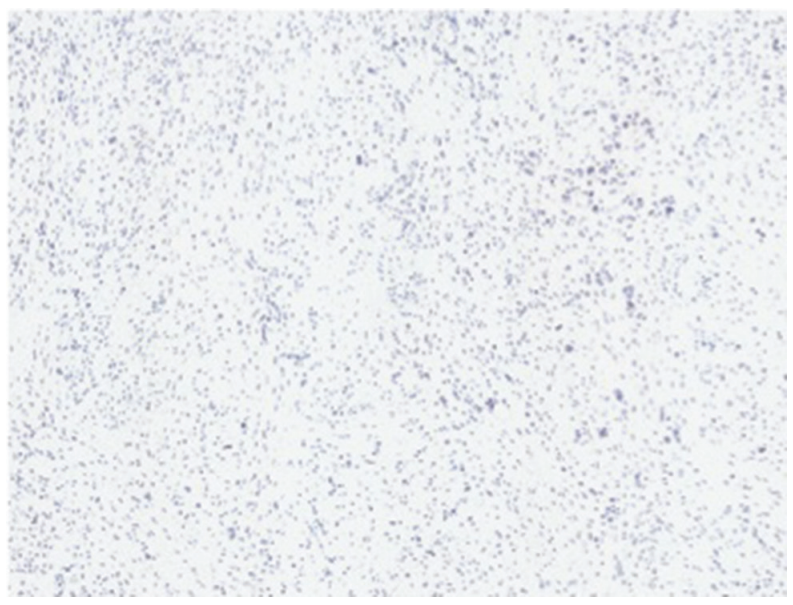

Fig. 6 Immunostaining of the surgical specimen with monoclonal antibody VE1 demonstrating negativity for the $B R A F$ V600E mutant protein. are positive for neuronal markers (e.g., synaptophysin, microtubule-associated protein 2 , neurofilament, and neuronal specific nuclear protein, NeuN) whilst the glial component, including Rosenthal fibers and eosinophilic granular bodies, may exist in various dimensions of structural characteristics (e.g., diffuse astrocytoma, pilocytic astrocytoma, and oligodendroglioma). These histological features may mistakenly lead to a diagnosis of astrocytoma. ${ }^{10)}$ In a report by Fiks et al., pathological reassessment using multiple neuronal markers identified $19 \%$ of previously diagnosed pilocytic astrocytoma patients as having gangliogliomas instead. ${ }^{11)}$

A $B R A F$ c.1799T $>$ A (V600E) mutation has been detected in $30-60 \%$ of gangliogliomas, which is predominantly expressed in the neuronal component. ${ }^{2)}$ In our case, $B R A F$ V600E mutant protein was not detected in the neuronal or glial components by VE1 immunohistochemistry. However, the BRAF V600E mutation has been identified in a small proportion of various types of glial tumors, including pilocytic astrocytomas and pleomorphic xanthoastrocytomas. Conversely, BRAF duplication/fusion events were more preferentially observed in cerebellar pilocytic astrocytomas in comparison to those in other regions. ${ }^{13)}$ Recently, the mutated $B R A F$ protein inhibitor, vemurafenib, has been used to treat $B R A F$ V600E mutation-positive metastatic or progressive melanomas. Rush et al. demonstrated the effect of vemurafenib in improving the symptoms, and markedly shrinking the size of the tumor, in a 13-year-old girl with a ganglioglioma of the brainstem. ${ }^{14)}$ Further investigations into the genetic background of gangliogliomas, therefore, could lead to elucidation of the pathogenesis of this rare neoplasm and pave the way for the development of new treatment modalities for unresectable or progressive lesions.

In conclusion, although gangliogliomas of the third ventricle are a rare occurrence, they should be included in the differential diagnosis of patients who present with hydrocephalus to ensure proper treatment strategies are in place before surgery. The pathogenesis of and genetic alternations in third ventricular gangliogliomas will need to be investigated in comparison with the various other glial neoplasms arising there.

Table 1 Reported gangliogliomas in the third ventricle

\begin{tabular}{|c|c|c|c|c|c|c|c|c|}
\hline $\begin{array}{l}\text { Case } \\
\text { no. }\end{array}$ & Author (year) & Age/sex & $\begin{array}{l}\text { Symptom } \\
\text { duration }\end{array}$ & Presentation & Treatment & Hydrocephalus & Hypopituitarism & Outcome \\
\hline 1. & Shono (2007) & $34 / \mathrm{F}$ & 3 months & Headache & TR & + & - & Unknown \\
\hline 2. & Shono (2007) & $52 / \mathrm{M}$ & 1 week & $\begin{array}{l}\text { Excessive thirst, nausea, appetite } \\
\text { loss, general fatigue }\end{array}$ & TR & - & + & $\begin{array}{l}\text { No recurrence } \\
\text { ( } 72 \text { months) }\end{array}$ \\
\hline 3. & Hauck (2008) & $20 / \mathrm{F}$ & Unknown & $\begin{array}{l}\text { Headache, blurred vision, } \\
\text { somnolence }\end{array}$ & TR & + & - & Unknown \\
\hline 4. & Deling (2013) & $15 / \mathrm{M}$ & 1 year & Headache, nausea, unsteady gait & GTR & + & - & $\begin{array}{l}\text { No recurrence } \\
\text { (19 months) }\end{array}$ \\
\hline 5. & Deling (2013) & $27 / \mathrm{M}$ & 2 years & Headache & TR & + & - & $\begin{array}{l}\text { No recurrence } \\
\text { (8 months) }\end{array}$ \\
\hline 6. & Present case & $38 / \mathrm{F}$ & 1 month & $\begin{array}{l}\text { Headache, nausea, insomnia, } \\
\text { pollakisuria }\end{array}$ & GTR & + & - & $\begin{array}{l}\text { No recurrence } \\
\text { (60 months) }\end{array}$ \\
\hline
\end{tabular}

F: female, GTR: gross total resection, M: male, TR: total resection. 


\section{Conflicts of Interest Disclosure}

The authors declared that they have no conflict of interest to report.

\section{References}

1) Shono T, Tosaka M, Matsumoto K, Onaka S, Yamaguchi S, Mizoguchi M, Iwaki T, Nakazato Y, Sasaki T: Ganglioglioma in the third ventricle: report on two cases. Neurosurg Rev 30: 253-258, 2007

2) Deling L, Nan J, Yongji T, Shuqing Y, Zhixian G, Jisheng W, Liwei Z: Intraventricular ganglioglioma prognosis and hydrocephalus: the largest case series and systematic literature review. Acta Neurochir (Wien) 155: 1253-1260, 2013

3) Samdani AF, Torre-Healy A, Khalessi A, McGirt M, Jallo GI, Carson B: Intraventricular ganglioglioma: a short illustrated review. Acta Neurochir (Wien) 151: 635-640, 2009

4) Zentner J, Wolf HK, Ostertun B, Hufnagel A, Campos MG, Solymosi L, Schramm J: Gangliogliomas: clinical, radiological, and histopathological findings in 51 patients. J Neurol Neurosurg Psychiatry 57: 14971502,1994

5) Doyle JB, Kernohan JW: Ganglioneuroma of the third ventricle with diabetes insipidus and hypopituitarism. J Nerv Ment Dis 73: 55-61, 1931
6) Anderson FM, Adelstein LJ: Ganglion cell tumor (ganglioglioma) in the third ventricle: operative removal with clinical recovery. Arch Surg 45: 129-139, 1942

7) Hauck EF, Vu L, Campbell GA, Nauta HJ: Intraventricular ganglioglioma. J Clin Neurosci 15: 1291-1293, 2008

8) Castillo M, Davis PC, Takei Y, Hoffman JC Jr: Intracranial ganglioglioma: MR, CT, and clinical findings in 18 patients. AJNR Am J Neuroradiol 11: 109-114, 1990

9) Rogojan L, Olinici CD: Ganglioglioma with glioblastoma component. Rom J Morphol Embryol 49: 403-406, 2008

10) Miller DC, Lang FF, Epstein FJ: Central nervous system gangliogliomas. Part 1: Pathology. J Neurosurg 79: 859-866, 1993

11) Fiks T, Jesionek-Kupnicka D, Zakrzewski K, Polis L, Liberski PP: Clinicopathological analysis of pilocytic astrocytomas and gangliogliomas in children. Folia Neuropathol 37: 152-156, 1999

12) Koelsche C, Wöhrer A, Jeibmann A, Schittenhelm J, Schindler G, Preusser M, Lasitschka F, von Deimling A, Capper D: Mutant BRAF V600E protein in ganglioglioma is predominantly expressed by neuronal tumor cells. Acta Neuropathol (Berl) 125: 891-900, 2013

13) Gessi M, Pietsch T: The diagnostic role and clinical relevance of determination of BRAF status in brain tumors. Personalized Medicine 10: 405-412, 2013

14) Rush S, Foreman N, Liu A: Brainstem ganglioglioma successfully treated with vemurafenib. J Clin Oncol 31: e159-160, 2013

Corresponding author:

Nayuta Higa, MD, Department of Neurosurgery, Graduate School of Medical and Dental Sciences, Kagoshima University, 8-35-1 Sakuragaoka, Kagoshima 890-8520, Japan.

$\triangle$ nayuhiga@gmail.com 